\title{
Photoisomerization Action Spectroscopy: Flicking the Protonated Merocyanine-Spiropyran Switch in the Gas Phase
}

\author{
Peter B. Markworth, Brian D. Adamson, Neville J. A. Coughlan, Lars Goerigk* and Evan J. \\ Bieske* $^{*}$
}

\section{Received Xth XXXXXXXXXX 20XX, Accepted Xth XXXXXXXXX 20XX}

First published on the web Xth XXXXXXXXXX 200X

DOI: 10.1039/b000000x

Laser spectroscopy and ion mobility spectrometry are combined to provide structural and photochemical information on photoisomerizing molecules in the gas phase. The strategy exploits the fact that an ion packet propelled through buffer gas by an electric field separates spatially and temporally into its constituent isomers because of small differences in their collision cross sections. Isomers selected by an electrostatic ion gate are exposed to wavelength tunable radiation, promoting formation of photoisomers that are separated in a second ion mobility stage. The approach is demonstrated for protonated merocyanine and spiropyran isomers formed through electrospray ionisation. Four isomers are observed whose relative abundances depend on pretreatment of the electrosprayed solution with either ultraviolet or visible light, and on collisional excitation before the ions are launched into the drift tube. The observations are interpreted in the light of accurate double-hybrid density functional theory calculations for the protonated spiropyran and merocyanine isomers that are used to predict structures, relative energies, isomerization barriers, collision cross sections and electronic absorption spectra. The two most abundant isomers, are merocyanine forms, in which the proton resides on the quinone oxygen atom, with either a trans or cis central bond in the linking polymethine chain. These two mero forms can be interconverted through photoexcitation, with different wavelength dependences for the forward and reverse photoisomerization processes. Protonated spiropyran is formed from protonated merocyanine isomers through collisional activation, but in only minor amounts through their photo-excitation over the $300-700 \mathrm{~nm}$ range.

\section{Introduction}

This paper explains how laser spectroscopy can be combined with ion mobility spectrometry (IMS) to provide information on the photoisomerization of charged molecules in the gas phase. The first part outlines the general strategy for measuring photo-induced changes in the collision cross sections for molecular ions drifting through a buffer gas, and describes its implementation using a tandem ion mobility spectrometer (IMS-photo-IMS machine) in which mobility-selected isomers are probed with laser beams. The second part of the paper describes the application of ion mobility methods to understanding the photochemistry of protonated spiropyran and merocyanine molecules in the gas phase. The photoiso-

+ Electronic Supplementary Information (ESI) available: Calculated relative electronic and Gibbs free energies for all isomers and barriers, calculated electronic excitation energies and oscillator strengths for all isomers, and images and Cartesian coordinates for all optimised structures.. See DOI: 10.1039/b000000x/

a School of Chemistry, The University of Melbourne, Melbourne, Australia. Tel: +61 38344 7082; Email: lars.goerigk@unimelb.edu.au, evanjb@unimelb.edu.au merization data are interpreted in the light of dispersioncorrected density functional theory (DFT) calculations for the protonated spiropyran and merocyanine molecules, which predict isomer structures, energies, and isomerization barriers. Accurate time-dependent double-hybrid DFT calculations are used to model electronic absorption spectra that are then compared with experimental photoisomerization action spectra of mobility-selected ions. The combined experimental and computational investigations provide new information on the stable structures and interconversion pathways of protonated spiropyran and merocyanine in the gas phase, and insights in the behaviour of these prototypical photoactive molecules in solution.

\section{Photoisomerization Action Spectroscopy of Molecular Ions}

After absorbing a photon, molecules are subject to a variety of fates including photon emission, internal conversion, intersystem crossing, photodissociation, electron 
emission, and photoisomerization. By exploiting these processes it is possible to implement sensitive detection strategies that form the basis of different "action" spectroscopic techniques. The action of choice for molecular ions has often been photodissociation. ${ }^{1-3}$ It's easy to understand why. Molecular ions can be mass selected and then exposed to a tuneable laser beam, with the photofragments separated and detected in a second mass selection step. The use of tandem mass spectrometry stages and detectors capable of sensing single ions, mean that the approach is both selective and extraordinarily sensitive. Target molecules with large dissociation energies that do not disintegrate following photon absorption can present problems, but these can be circumvented by tagging the ions with rare gas atoms ( $\mathrm{Ar}$ or $\mathrm{He}$ ), which are easily dislodged following photo excitation, or alternatively by unleashing intense light beams that promote multiphoton dissociation. The advent of convenient table-top infrared (IR), visible and ultraviolet (UV) optical parametric oscillators (OPOs), and also free electron lasers, often coupled with modified commercial mass spectrometers with electrospray ion sources, has opened up the field in the past 10 years. ${ }^{4,5}$

Despite progress, there are certain classes of charged molecules and molecular processes that are difficult to characterise using a straightforward photodissociation approach. For example, many molecular ions have several different isomeric forms whose infrared and electronic spectra may be subtly different (e.g. the various isomers of retinal ${ }^{6}$ ) or alternatively have vastly different structures, and vibrational and electronic spectra (e.g. spiropyrans, fulgides and diarylethenes). Disentangling spectra of coexisting isomers is often problematic and for charged molecules has normally been accomplished using double resonance and hole burning photodissociation strategies, usually in conjunction with quantum chemical predictions for vibrational frequencies and intensities. ${ }^{7-9}$

Recently, we have developed an alternative approach for spectroscopically investigating charged molecular isomers and exploring photoisomerization processes in the gas phase. The method is based on ion mobility spectrometry (IMS), a technique whereby charged molecules are separated as they drift through a buffer gas under the influence of an electric field (Fig. 1). An ion's collision cross section and velocity depend on its geometry - compact, folded ions drift more rapidly than open, extended ions. Exposing drifting ions to laser light with an appropriate wavelength leads to photoisomerization, changing their collision cross section and drift velocity. This forms the basis of an action technique we call photoisomerization action (PISA) spectroscopy. In our original investigations,

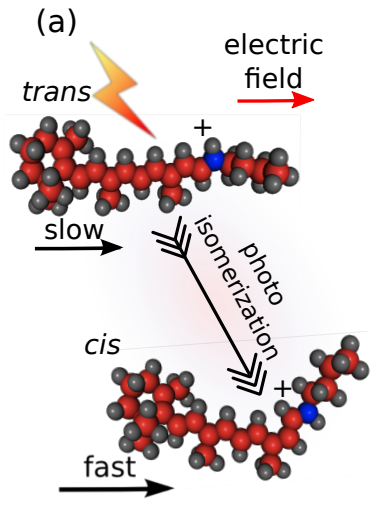

(b)

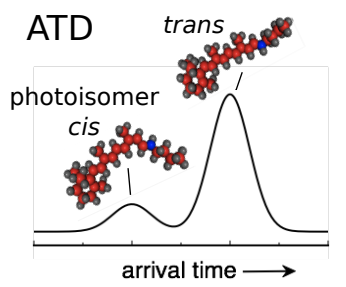

(c)

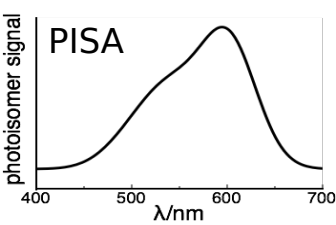

Fig. 1 (a) Photoisomerization changes the drift speed of a molecular ion propelled by an electric field through buffer gas. (b) The photo-isomer appears as a distinct peak in the arrival time distribution (ATD). (c) A photoisomerization action (PISA) spectrum is obtained by measuring the intensity of the photo-isomer peak as a function of wavelength.

we exposed a packet ions to a tunable laser beam as they entered the drift region of the IMS, monitoring laser induced changes in the ions' arrival time distribution. This photo-IMS approach was used to probe the photoisomerization of carbocyanine dyes, ${ }^{10,11}$ the retinal protonated Schiff base (RPSB), ${ }^{12}$ and also to elucidate the structure of $\beta$-ionone photofragments from RPSB. ${ }^{13}$ More recently, we have adopted a tandem IMS-photo-IMS arrangement, so that a particular isomer can be selected in a first IMS stage and then irradiated with tunable light, with the photoisomers separated in a second IMS stage. ${ }^{14}$ The tandem IMS-photo-IMS approach has the advantage that the target isomer is selected before being irradiated and that the product isomers can also be identified. Another advantage is that the photoisomer signal is easily distinguished from the parent ion signal from which it is separated temporally. Because the photoisomer ions are observed against zero background, photoisomerization action spectra can be recorded with high sensitivity. In this paper we describe the IMS-photo-IMS strategy, demonstrating its use for providing spectroscopic, structural, and photochemical information on molecular ions in the gas phase, using protonated spiropyran/merocyanine molecules as an example.

Using IMS to derive structural information on gasphase ions has a long history. An excellent account of IMS development is given in Ref. 15, while refs. 16-19 provide good reviews of IMS methods and applications. Once the province of specialist laboratories with purposebuilt machines, ${ }^{20-24}$ IMS has become much more widely 
adopted with the release of commercial instruments with reliable interfaces to electrospray ion sources and mass spectrometers. ${ }^{25}$ Deploying tandem IMS to follow structural changes in molecular ions was pioneered by Clemmer and coworkers who developed a machine in which a particular isomer was selected in a first IMS stage followed by collisional activation and separation of product isomers in a second IMS stage. ${ }^{26-28}$ Our IMS-photo-IMS machine operates on a similar principal with the collision zone replaced by a photoisomerization zone.

Recently, several groups have combined ion mobility spectrometry with fixed-wavelength laser excitation to probe photofragmentation of mobility-selected molecular ions. For example, Misaizu and coworkers used a drift cell IMS to select linear or cyclic carbon clusters $\left(\mathrm{C}_{n}^{+}, n=7\right.$ 10) before photodissociation with pulsed $355 \mathrm{~nm}$ radiation in a time-of-flight mass spectrometer, finding different photofragment branching ratios according to whether linear or cyclic $\mathrm{C}_{n}^{+}$isomers were excited. ${ }^{29}$ Clemmer, Reilly and coworkers, selected oligosaccharide isomers using a drift tube IMS that were then fragmented with the $157 \mathrm{~nm}$ output from an excimer laser, and identified a dependence of the photofragment distribution on the selected isomer. ${ }^{30}$ Warnke $e t$ al.used a similar approach to separate isomers of protonated ubiquitin cations, 31 again discovering a distinct isomer specificity for the photofragmentation pattern. Bellina et al. reported UV photodissociation mass spectra for mobility-selected flavin ions. ${ }^{32}$

Infrared and electronic spectra of mobility-selected ions have also been obtained using resonance enhanced photodissociation. Among several studies, Hernandez et al. employed a differential ion mobility spectrometer (DIMS) to separate saccharides ionized with $\mathrm{Li}^{+}$, prior to an IR photodissociation stage, and were able to identify distinct IR spectra for hydrated $\alpha$ and $\beta$ anomers. ${ }^{33}$ Papadopoulos et al. separated isomers of doubly protonated bradykinin using field-asymmetric waveform ion mobility spectrometry (FAIMS), before a resonance enhanced photodissociation stage in a cryogenic ion trap. ${ }^{34}$ With judicious tuning of FAIMS parameters, photofragmentation spectra could be recorded for the two dominant isomers, although care was necessary to prevent isomeric mixing after FAIMS separation and before the laser probing zone. Warnke et al. separated two isomers of protonated benzocaine using using drift tube IMS, and probed each isomer using IR light from a free electron laser, which induced photodissociation. Distinct IR spectra were obtained for $\mathrm{N}$ - and O-protonated isomers. ${ }^{35}$ The aforementioned investigations have all relied on measuring the photodissociation response of isomer-selected ions, in contrast to the IMS-photo-IMS approach which depends on detecting a change in the molecular collision cross section following photoexcitation.

\section{IMS-photo-IMS Apparatus}

In our investigations, photoisomerizing ions are probed using a purpose-built ion mobility apparatus. ${ }^{10-12,14}$ As shown in Fig. 2, the machine is essentially a tandem drifttube IMS-IMS machine with provision for a laser beam to overlap the drifting ions between the two IMS regions. The ions, which are created using electrospray source, pass through a heated transfer capillary and accumulate in an ion funnel (IF1). An electrostatic ion gate (IG1) is opened periodically (at $20 \mathrm{~Hz}$ ) to inject $100 \mu$ s ion bunches into the drift section filled with $\mathrm{N}_{2}$ gas ( $P=5-10$ Torr) and with a $44 \mathrm{~V} / \mathrm{cm}$ drift field sustained by a series of ring electrodes. A Bradbury-Nielsen ion gate (IG2) approximately half way along the drift tube can be opened for $100 \mu \mathrm{s}$ at an appropriate delay with respect to the first gate (IG1) to pass ions with a particular drift speed (i.e. collision cross section), whereas slower and faster ions are blocked. Transmitted ions are irradiated $15 \mathrm{~mm}$ after IG2 with an appropriately timed light pulse from a tuneable optical parametric oscillator (OPO, $5 \mathrm{~ns}$ pulse width, bandwidth $8 \mathrm{~cm}^{-1}$, scan range $300-700 \mathrm{~nm}$ ). The light beam typically has a cross section of $4 \mathrm{~mm} \times 6 \mathrm{~mm}$ and energy of $0.5-3 \mathrm{~mJ} /$ pulse. A photo-induced change in ion conformation and drift mobility results in separation of parent and photoisomer ions over the remaining section of the drift region.

After the drift region, ions are collected by a second ion funnel and pass through a $0.35 \mathrm{~mm}$ orifice into an octopole ion guide, then a quadrupole mass filter, and are finally sensed by a channeltron ion detector connected to a multichannel scaler. By these means, a histogram is built up representing the ions' arrival time distribution (ATD), which typically exhibits peaks corresponding to different isomers. When operated with only the first ion gate (IG2 open), the IMS has a mobility resolution of $t_{d} / \Delta t_{d} \approx 80$. When used in IMS-photo-IMS mode, the first and second stages each have a mobility resolution of $\approx 50$. Ion count rates, with IG1 pulsed at $20 \mathrm{~Hz}$ and $100 \mu$ s opening time, are $100-5000 \mathrm{~s}^{-1}$, depending on electrospray conditions and the particular ion being studied.

Because of small fluctuations in the ions' flight times (due mainly to minor pressure changes), a routine is incorporated into the data collection program that adjusts the timings for opening IG2 and firing the OPO. Approximately every 10 minutes, IG2 is opened completely so that the entire ATD can be recorded. The position of a prominent ATD peak is used to determine optimum timing for opening IG2 to transmit the target isomer, and

This journal is @ The Royal Society of Chemistry [year]

Journal Name, 2010, [vol], 1-14 | 3 


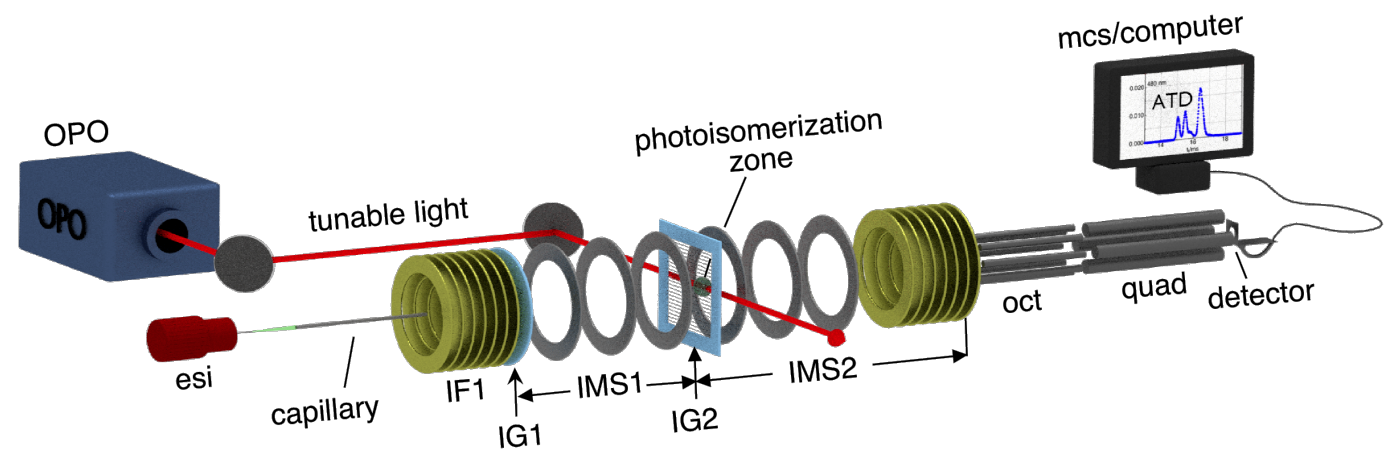

Fig. 2 Ion mobility spectrometer for probing photoisomerization of charged molecules. Electrosprayed ions pass through a heated desolvation capillary and into an ion funnel (IF1) from where they are injected through an electrostatic gate (IG1) into the drift region ( $\mathrm{N}_{2}$ gas, $P=5-10$ Torr, drift field $\approx 44 \mathrm{~V} / \mathrm{cm}$ ). After travelling $45 \mathrm{~cm}$, the ions meet a second ion gate (IG2) that can be opened to transmit the target isomer. Shortly after IG2 the ions are exposed to light from an OPO. After passing through the drift tube, ions are collected by a second ion funnel (IF2), travel through an octopole ion guide, and are mass selected by a quadrupole mass filter.

appropriate delay for triggering the pulsed OPO. This routine helps maintain temporal overlap between the target ions and the laser pulse during longer photoisomerization scans, which can last for up to 4 hours.

Currently, it is difficult to probe drifting ions with shorter wavelength UV light $(\lambda \leq 310 \mathrm{~nm})$ because background ions, formed through multiphoton ionisation of impurities in the background gas, tend to arrive at the same time as target ions generated by the electrospray ionization source.

\subsection{Drift times, mobilities, collision cross sections, and photoisomerization}

In the low field regime, an ion's drift velocity $\left(v_{d}\right)$ is proportional to the applied electric field $(E),{ }^{36}$

$$
v_{d}=K \times E,
$$

where $K$, the ion's mobility, is given by the Mason-Schamp equation,

$$
K=\frac{3 z e}{16 N} \sqrt{\frac{2 \pi}{\mu k_{B} T}}\left(\frac{1}{\Omega^{(1,1)}(T)}\right) .
$$

Here, $z$ is number of elementary charges carried by the ion, $e$ is the electronic charge, $N$ is the number density of the bath gas, $\mu$ is the reduced mass of the colliding ion-neutral pair, $k_{B}$ is the Boltzmann constant and $T$ is the absolute temperature. $\Omega^{(1,1)}(T)$ is the orientationally averaged collision integral, which depends on the intermolecular potential energy surface for the interaction between the neutral buffer gas and the ion, and so is sensitive to the molecular structure.
In the IMS-photo-IMS machine, ions travel through several regions, which have different lengths $\left(l_{i}\right)$ and voltage drops $\left(V_{i}\right)$, as they pass from the first ion gate (IG1) to the detector (drift region from IG1 to IG2, drift region from IG2 to IF2 and through IF2). The time to travel across one of the drift regions is:

$$
t_{i}=\frac{l_{i}^{2}}{K V_{i}},
$$

so that the transit drift time is:

$$
t_{\text {total }}=\sum_{i} \frac{l_{i}^{2}}{K V_{i}}+t_{\text {oct }}+t_{\text {quad }}
$$

where $t_{\text {oct }}$ and $t_{\text {quad }}$ are the passage times through the octopole and quadrupole regions, which are at pressures $2 \times 10^{-4}$ Torr and $10^{-6}$ Torr, respectively, and through which the ions pass essentially unimpeded by collisions. Therefore, the mobility $K$ can be related to total transit time between IG1 and the detector.

$$
K=\frac{1}{t_{\text {total }}-t_{\text {oct }}-t_{\text {quad }}} \sum_{i} \frac{l_{i}^{2}}{V_{i}}
$$

Note, that the flight times through the octopole and quadrupole are relatively short $(\approx 100 \mu \mathrm{s})$ compared to transit times through the drift region $(5-30 \mathrm{~ms})$. The mobility $K$ can be used to calculate the collision cross section using Eqn. 2. Generally, cross sections measured with our instrument match literature values. For example, for tetrabutyl ammonium (TBA) in $\mathrm{N}_{2}$ we find $\Omega=171.6 \AA^{2}$ compared to $\Omega=166.6 \AA^{2}$ from ref. 37 .

When the instrument is used in tandem IMS-photoIMS mode, photoisomerization occurs shortly after IG2 
and the parent and daughter ions are separated in the second half of the drift tube, arriving at the detector at slightly different times. Using Eqn. 4 it is possible to show that the difference in the parent and daughter arrival times is given by:

$$
\Delta t=\left(\frac{1}{K_{P}}-\frac{1}{K_{D}}\right) \sum_{j} \frac{l_{j}^{2}}{V_{j}},
$$

where the sum refers to regions after the isomerization zone (i.e. the remainder of the drift region and IF2) and $K_{P}$ and $K_{D}$ are the parent and daughter ion mobilities, respectively. Using Eqn. 6 it is possible to calculate the daughter photoisomer's mobility, and with Eqn. 2, the collision cross section. In our IMS-photo-IMS machine, the photoisomerization region lies approximately halfway along the drift tube, so that if the ion population consists of two isomers $A$ and $B$ with arrival times $t_{A}$ and $\mathrm{t}_{B}$, respectively, then if $A$ is transformed to $B$ in the photoisomerization region, the photo-isomer peak arrives approximately at $\left(t_{A}+t_{B}\right) / 2$.

\section{Protonated Spiropyran/Merocyanine}

In the remainder of the paper, we show how the photoIMS approach can be used to elucidate the photochemical behaviour of the protonated spiropyran/merocyanine system. Spiropyrans are a class of photoactive molecules that have found roles as molecular switches, thermal and chemical sensors, and as photo-induced proton transfer components in photoelectric cells. ${ }^{38,39}$ In this paper we focus on 1',3',3'-trimethyl-6-nitrospiro[chromene-2,20indoline] (SP). As shown in Figure 3 (upper left), the neutral spiropyran (SP) molecule consists of indoline and chromene subunits sharing a spiro carbon atom. Absorption of an ultraviolet photon converts SP to the transitory cis merocyanine (MC) isomer and then rapidly to the open trans, merocyanine (MC) isomer, which due to its extended, conjugated structure absorbs visible light. The MC form potentially has $2^{3}=8$ isomers according to whether each of the $\alpha, \beta$ and $\gamma \mathrm{C}-\mathrm{C}$ bonds has trans (T) or cis (C) conformation (Fig. 3, upper right). Note that in the following discussion if a bond could either be trans or cis, we label its configuration $\mathrm{X}$. The lowest energy isomer for MC is predicted to be the TTC isomer, followed by the TTT, CTC and CTT isomers. ${ }^{40-42}$ The process can be reversed by either heating $\mathrm{MC}$ or exposing it to visible light. Experimentally, conformational transformations of the SP/MC system have been studied using femtosecond pump-probe spectroscopy, ${ }^{43}$ femtosecond UV/mid-IR spectroscopy, ${ }^{44}$ and femtosecond transient absorption spectroscopy. ${ }^{45}$ Electron diffraction of photoisomerized
SP in the gas phase showed preferential formation of the CTC isomer of MC. ${ }^{46}$

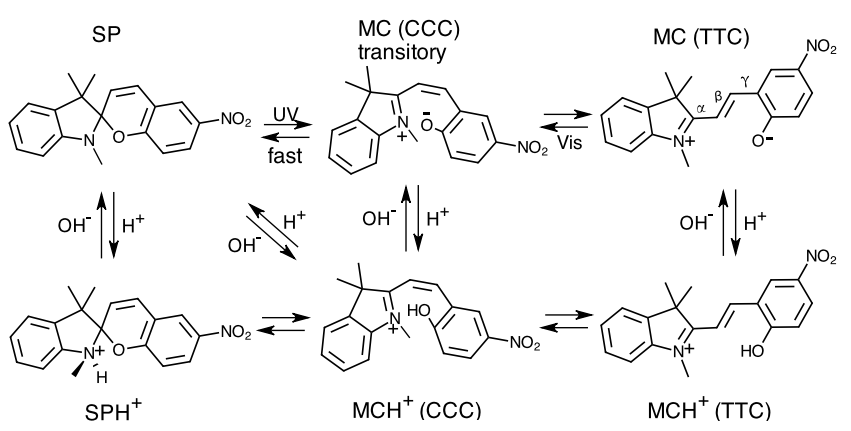

Fig. 3 Structures of neutral and protonated spiropyran and merocyanine molecules with a simplified scheme showing their interconversion through acid-base equilibria. Further details of the transformations are discussed in Section 6.

The photo- and thermo-chromic behaviour of the neutral SP/MC system is altered in acidic solutions with $\mathrm{XTX} \mathrm{MCH}^{+}$isomers with a protonated $\mathrm{O}$ atom becoming the most stable forms (Fig. 3, lower right). ${ }^{47}$ Computational studies show that although SP can be protonated on the $\mathrm{N}$ atom to form a stable $\mathrm{SPH}^{+}$species, protonation on the $\mathrm{O}$ atom leads to barrierless ring opening to form the $\mathrm{CCC} \mathrm{MCH}^{+}$isomer. ${ }^{42}$ There is clear evidence from $\mathrm{UV} /$ Vis and NMR spectra for SP, MC, and trans $\mathrm{MCH}^{+}$ in solution. Furthermore, NMR studies implicate XCX $\mathrm{MCH}^{+}$with a cis $\beta$ bond as an intermediate in the acid catalysed racemization of SP. The proposed mechanism involves ring-opening initiated by protonation of the $\mathrm{O}$ atom in SP, formation of XCX MCH ${ }^{+}$, followed by internal rotation about the single $\alpha$ bond in the polyene chain, deprotonation and ring closure to form the other SP enantiomer. ${ }^{48}$ Unfortunately, it is difficult to monitor $\mathrm{SPH}^{+}$ and cis $\mathrm{MCH}^{+}$in solution using $\mathrm{UV} /$ Vis spectroscopy. As shown below, $\mathrm{SPH}^{+}$absorbs at $220 \mathrm{~nm}$ in an inconvenient spectral region to probe in solution, whereas XCX $\mathrm{MCH}^{+}$isomers absorb near $340 \mathrm{~nm}$ making them difficult to distinguish from neutral SP, which absorbs in the same region. ${ }^{38}$

The existence of several isomers of the protonated spiropyran/merocyanine system in the gas phase was confirmed by Rogers et al. who used ion mobility spectrometry to investigate $\mathrm{SPH}^{+} / \mathrm{MCH}^{+}$ions electrosprayed from solution. ${ }^{49}$ Despite the large number of potential isomers, only 3 peaks were observed in the arrival time distribution, the slowest of which was assigned to an extended XTX $\mathrm{MCH}^{+}$form, whereas the faster two peaks were assigned to more compact $\mathrm{XCX} \mathrm{MCH}^{+}$and $\mathrm{SPH}^{+}$ isomers (without firm attribution of either peak to a spe- 
cific isomer).

Here we build on the earlier IMS investigation by probing the photo- and collision induced transformations of $\mathrm{SPH}^{+} / \mathrm{MCH}^{+}$ions in the gas phase using the IMS-photo-IMS approach. We extend the previous work in several ways. First, our IMS apparatus has higher mobility resolution than the one used by Rogers et al., ${ }^{49}$ allowing us to find evidence for an additional isomer. Second, we have photoisomerized mobility-selected isomers in the drift region to map photoisomerization pathways, and measure photoisomerization action spectra. Third, additional information on isomerization pathways is gathered by collisionally activating $\mathrm{SPH}^{+} / \mathrm{MCH}^{+}$ions in the ion source region, monitoring the transformation of isomer populations. Finally, we have undertaken dispersion-corrected density functional theory (DFT) calculations for the protonated SP/MC conformers, identifying stable structures, isomerization barriers, collision cross sections, and, using time-dependent DFT, electronic absorption spectra. Our computational analysis differs from previous studies on the protonated SP/MC system in two ways. A previous investigation of the protonated system was carried out for a derivative containing a long side chain attached to the nitrogen atom, ${ }^{42}$ whereas we consider the simpler system with a methyl group as the substituent. Therefore, we avoid dealing with complicating side-chain conformations. Furthermore, the quantumchemical methods used in the present study should be more accurate and reliable than those used in previous computational studies on the protonated and neutral systems. ${ }^{40-42}$ Therefore, our joint theoretical and experimental investigations should provide a more comprehensive picture of the properties and photophysical behaviour of the $\mathrm{SPH}^{+} / \mathrm{MCH}^{+}$system than hitherto available.

\subsection{Quantum-chemical calculations for $\mathrm{SPH}^{+} / \mathrm{MCH}^{+}$}

To characterise the isomeric landscape of the $\mathrm{SPH}^{+} / \mathrm{MCH}^{+}$ system we conducted DFT calculations for $4 \mathrm{SPH}^{+}$isomers and $8 \mathrm{MCH}^{+}$isomers. All DFT calculations were carried out with ORCA 3.0.2. ${ }^{50}$ Geometries and zeropoint-energy ( $\mathrm{ZPE})$ corrections were obtained at the dispersion corrected PW6B95 ${ }^{51}$-D3(BJ) ${ }^{52,53} /$ def2-TZVP 54 level of theory. Subsequent single-point calculations were carried out at the dispersion-corrected double-hybrid DSD-PBEP86-D3(BJ) ${ }^{55} /$ def2-QZVP ${ }^{54}$ level and the results were combined with the previously obtained ZPE values. Double-hybrid density functionals are combinations of conventional hybrid functionals with secondorder Møller-Plesset perturbative electron-correlation contributions, and thus they belong on a higher (and more accurate) rung on Perdew's Jacob's Ladder scheme ${ }^{56}$ than conventional hybrids. ${ }^{57,58}$

Comprehensive benchmark studies with the large GMTKN30 59,60 database for general main-group thermochemistry, kinetics and noncovalent interactions, ${ }^{60,61}$ as well as numerous successful applications (see Ref. 58 for a review), support the conclusion that dispersioncorrected double-hybrids offer higher accuracy and robustness than commonly used global or range-separated hybrid functionals, such as B3LYP ${ }^{62,63}$ or CAM-B3LYP, ${ }^{64}$ used in previous computational studies on the SP/MC system. ${ }^{40-42}$ In particular, the functional chosen herein is known to provide reliable isomerization energies and barrier heights. $55,58,65$ The average error of DSD-PBEP86D3(BJ) for main-group thermochemistry has been determined to be around $5 \mathrm{~kJ} / \mathrm{mol}$, ${ }^{58}$ whereas average errors of dispersion-corrected B3LYP and CAM-B3LYP have been shown to be about 15.5 and $14.5 \mathrm{~kJ} / \mathrm{mol}$, respectively. ${ }^{61}$

Double-hybrid functionals also outperform other approaches, including common wave-function methods, in the description of vertical electronic transition energies of organic chromophores ${ }^{66-69}$ and prediction of absorption spectra. ${ }^{66,70}$ The current implementation in ORCA does not support full linear-response time-dependent DFT (TD-DFT) calculations with double-hybrids. Instead it employs the much simpler Tamm-Dancoff approximation (TDA-DFT) ${ }^{71,72}$ This approximation, however, is not expected to influence the excitation energies significantly. ${ }^{66}$ Herein, we report results for the B2GP-PLYP ${ }^{73}$ functional at the def2-TZVP level, which has an average accuracy of $0.14 \mathrm{eV}$ for low-lying valence transitions in similar chromophores, compared to errors of $0.3 \mathrm{eV}$ or more for standard density functionals, such as B3LYP. ${ }^{68}$ UV/Vis spectra were simulated by overlapping Gaussian functions for each transition, with a value of $\sigma=0.2 \mathrm{eV}$ for the width of the absorption band at a height of $1 / e$.

Based on the DFT geometries we calculated collision cross sections, mobilities and arrival times for the $\mathrm{SPH}^{+}$ and $\mathrm{MCH}^{+}$isomers using the MOBCAL program with the trajectory method. ${ }^{74,75}$ Atom-atom potential energy parameters for the interaction between $\mathrm{N}_{2}$ and the colliding molecule were taken from ref. 25. Partial atomic $\left(\mathrm{CHELPG}^{76}\right)$ charges were derived from the electrostatic potential of each system at the same level of theory as the geometry optimizations. Note that the predicted collision cross sections are based on crude intermolecular potential energy surfaces (sum of atom-atom potential energy functions) and probably only serve as an approximate guide to the actual collision cross sections. 
Table $1 \mathrm{DFT}$ results for $\mathrm{SPH}^{+}$and $\mathrm{MCH}^{+}$isomers. Diagonal elements are isomer energies $(\mathrm{kJ} / \mathrm{mol})$ with respect to that of the TTT isomer of $\mathrm{MCH}^{+}$. Blue off-diagonal elements are isomerization barriers for rotation around single bonds in $\mathrm{MCH}^{+}$or inversion of the five-membered ring in $\mathrm{SPH}^{+}$. Yellow cells are estimated barriers for isomerization around the central double bond in $\mathrm{MCH}^{+}$. Red cells show proton-exchange barriers between $\mathrm{MCH}^{+}$and $\mathrm{SPH}^{+}(\mathrm{O}$ and $\mathrm{N}$ protonation $)$. All values are $\mathrm{ZPE}$-corrected electronic energies obtained at DSD-PBEP86-D3(BJ)/def2-QZVP (upper row in each cell) and PW6B95-D3(BJ)/def2-TZVP (lower row) levels of theory. The last column gives each isomer's calculated collision cross section with $\mathrm{N}_{2}$.

\begin{tabular}{|c|c|c|c|c|c|c|c|c|c|c|c|c|c|}
\hline & TTT & TTC & CTT & СТC & CCT & TCT & TCC & $\mathrm{CCC}$ & $\mathrm{SP}(\mathrm{S})_{1}$ & $\mathrm{SP}(\mathrm{S})_{2}$ & $\mathrm{SP}(\mathrm{R})_{1}$ & $\mathrm{SP}(\mathrm{R})_{2}$ & $\begin{array}{l}\Omega \\
\AA^{2}\end{array}$ \\
\hline TTT & $\begin{array}{l}0.0 \\
(0.0)\end{array}$ & $\begin{array}{l}35.7 \\
(41.5)\end{array}$ & $\begin{array}{l}26.9 \\
(33.4)\end{array}$ & & & $\begin{array}{c}- \\
(168.8)\end{array}$ & & & & & & & 187.4 \\
\hline TTC & $\begin{array}{l}34.0 \\
(37.7)\end{array}$ & $\begin{array}{l}1.7 \\
(3.8)\end{array}$ & & $\begin{array}{l}23.2 \\
(28.7)\end{array}$ & & & $\begin{array}{c}- \\
(175.3)\end{array}$ & & & & & & 189.3 \\
\hline CTT & $\begin{array}{l}21.2 \\
(26.5)\end{array}$ & & $\begin{array}{l}5.7 \\
(6.9)\end{array}$ & $\begin{array}{l}31.5 \\
(37.1)\end{array}$ & $\begin{array}{c}- \\
(164.5)\end{array}$ & & & & & & & & 188.3 \\
\hline CTC & & $\begin{array}{l}16.1 \\
(21.2)\end{array}$ & $\begin{array}{l}28.4 \\
(32.7)\end{array}$ & $\begin{array}{l}8.8 \\
(11.3)\end{array}$ & & & & $\begin{array}{c}- \\
(173.1)\end{array}$ & & & & & 189.0 \\
\hline CCT & & & $\begin{array}{c}- \\
(150.5)\end{array}$ & & $\begin{array}{l}12.0 \\
(20.9)\end{array}$ & & & $\begin{array}{l}19.7 \\
(21.1)\end{array}$ & & & & & 173.8 \\
\hline TCT & $\begin{array}{c}- \\
(139.8)\end{array}$ & & & & & $\begin{array}{l}19.7 \\
(29.0)\end{array}$ & $\begin{array}{l}31.9 \\
(31.3)\end{array}$ & & & & & & 179.8 \\
\hline TCC & & $\begin{array}{c}- \\
(141.3)\end{array}$ & & & & $\begin{array}{l}22.1 \\
(22.5)\end{array}$ & $\begin{array}{l}29.5 \\
(37.8)\end{array}$ & & & & & & 184.4 \\
\hline CCC & & & & $\begin{array}{c}- \\
(158.7)\end{array}$ & $\begin{array}{l}12.5 \\
(16.3)\end{array}$ & & & $\begin{array}{l}19.2 \\
(25.7)\end{array}$ & $\begin{array}{l}164.1 \\
(178.6)\end{array}$ & & & & 183.1 \\
\hline $\mathrm{SP}(\mathrm{S})_{1}$ & & & & & & & & $\begin{array}{l}132.8 \\
(133.7)\end{array}$ & $\begin{array}{l}50.5 \\
(70.6)\end{array}$ & $\begin{array}{l}17.1 \\
(15.8)\end{array}$ & & & 184.5 \\
\hline $\mathrm{SP}(\mathrm{S})_{2}$ & & & & & & & & & $\begin{array}{l}26.0 \\
(25.6)\end{array}$ & $\begin{array}{l}41.6 \\
(60.8)\end{array}$ & & & 183.2 \\
\hline $\mathrm{SP}(\mathrm{R})_{1}$ & & & & & & & & & & & $\begin{array}{l}48.3 \\
(69.1)\end{array}$ & $\begin{array}{l}12.7 \\
(10.9)\end{array}$ & 184.9 \\
\hline $\mathrm{SP}(\mathrm{R})_{2}$ & & & & & & & & & & & $\begin{array}{l}12.2 \\
(10.9)\end{array}$ & $\begin{array}{l}48.8 \\
(69.1)\end{array}$ & 182.1 \\
\hline
\end{tabular}

\section{Results}

\subsection{Calculated isomers and isomerization barriers}

The diagonal elements of Table 1 are zero-point-energy (ZPE) corrected electronic energies of the $8 \mathrm{MCH}^{+}$isomers relative to the all-trans (TTT) isomer (see structure in Fig. 4). The results for the dispersion-corrected doublehybrid functional DSD-PBEP86-D3(BJ) reveal that the energy difference between the TTT and TTC isomers is only $1.7 \mathrm{~kJ} / \mathrm{mol}$ and, thus, below the aforementioned average accuracy of $5 \mathrm{~kJ} / \mathrm{mol}$ for this functional. Therefore we cannot definitely conclude which of these two isomers is more stable and for the remainder of this discussion we assume that they are energetically equivalent. While all $\mathrm{MCH}^{+}$isomers with a central trans bond (XTX) have relative energies below $9 \mathrm{~kJ} / \mathrm{mol}$ with respect to TTT, all XCX isomers lie between $12 \mathrm{~kJ} / \mathrm{mol}$ (CCT) and $29.5 \mathrm{~kJ} / \mathrm{mol}$ (TCC) higher in energy. This is caused by additional steric crowding between the two ends of the molecule. The CCC isomer, which conceivably plays a role in the formation of the $\mathrm{SPH}^{+}$, lies $19 \mathrm{~kJ} / \mathrm{mol}$ higher in energy than the TTT form and due to steric crowding it assumes a helical shape (Fig. 4).

The blue off-diagonal elements of the matrix in Table 1 show selected barriers between various $\mathrm{MCH}^{+}$isomers for internal rotation about either the $\alpha$ or the $\gamma$ bond (see Fig. 3). The barriers were identified with the help of relaxed surface scans for the rotation around the bond of interest, followed by a standard transition state optimization. The rotation profiles for the $\alpha$ and $\gamma$ torsion angles did not reveal the involvement of any conical in- 
tersections and we conclude that the relevant bonds are mainly of $\sigma$ character. Most rotation barriers lie between 20 and $36 \mathrm{~kJ} / \mathrm{mol}$, with the highest barrier being between the TTT and TTC isomers $(35.7 \mathrm{~kJ} / \mathrm{mol})$. The lowest barrier is for conversion of CCC to CCT $(12.5 \mathrm{~kJ} / \mathrm{mol})$. Note that due to steric hindrance the rotation of one end of the merocyanine can also induce rotation of the opposite end group making it difficult to identify the isomerization barriers between all of the merocyanine isomers. Considering such simultaneous rotations around two bonds is beyond the scope of this study and is not considered further.

As expected, the energy profile for rotation around the central double bond $(\beta)$ reveals the presence of conical intersections and single reference methods are known to be inadequate for describing these correctly. Therefore, the values given in Table 1 (yellow cells) are only an estimate to the thermal barrier at a dihedral angle $\beta$ of $90^{\circ}$. Moreover, due to high-spin contamination, the usage of the unrestricted perturbative contribution is problematic for double-hybrid functionals. ${ }^{77,78}$ To provide a rough estimate for this barrier, we use an unrestricted treatment with the PW6B95-D3(BJ) hybrid functional and values for this method are shown in parentheses in Table 1. Overall, PW6B95-D3(BJ) is known to be one of the most robust and accurate hybrid functionals and although its average accuracy is lower than that of double-hybrids, ${ }^{61}$ the overall trends for the isomerization energies and barriers in Table 1 are very similar. Thus, this functional should be sufficient for our purposes. The calculated double bond barriers are between 140 and $175 \mathrm{~kJ} / \mathrm{mol}$, and thus significantly higher than the barriers for rotation around the single bonds.
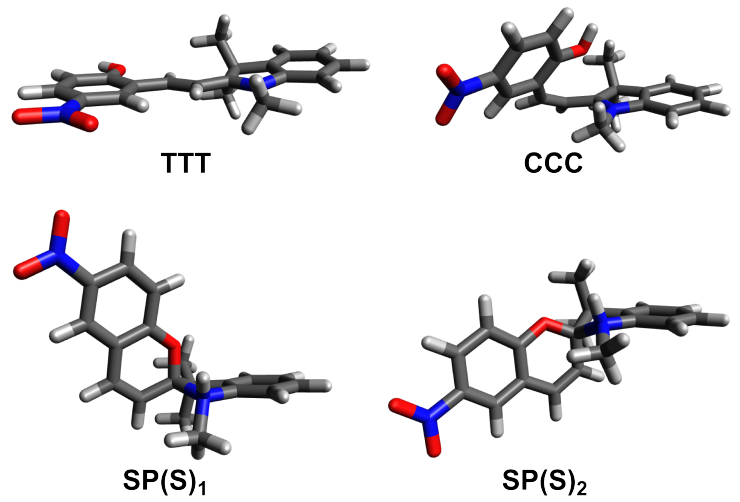

Fig. 4 Structures of two $\mathrm{MCH}^{+}$isomers (TTT and CCC) and two $\mathrm{SPH}^{+}$conformers $\left(\mathrm{SP}(\mathrm{S})_{1 / 2}\right)$ obtained at the PW6B95-D3(BJ)/def2-TZVP level of theory.
Table 1 also provides energies of the spiropyran isomers relative to the TTT merocyanine form. Our results confirm previous findings that protonated spiropyrans are less stable than the merocyanines ${ }^{42}$ - by up to $50 \mathrm{~kJ} / \mathrm{mol}$ according to our calculations. The protonated spiropyran has two stereogenic centres in the fivemembered ring. If we consider the $\mathrm{sp}^{3}$-hybridized carbon atom to be in $R$ configuration, then two diastereoisomers are possible, with the nitrogen atom being either in $S$ or $R$ configuration. In Table 1 both possibilities are shown and dubbed SP(S) or SP(R), respectively. Furthermore, we also note that the five-membered ring can assume two different envelope conformations due to inversion of the indoline ring. The two conformers for SP(S) are shown in Fig. 4 and the relevant inversion barrier heights are included in Table 1.

Finally, Table 1 also lists the barrier heights for proton transfer between the SP(S) spyropyran and the CCC merocyanine forms; the corresponding transition-state structure is shown in the electronic supplementary information (ESI). The barrier the proton needs to overcome to migrate from the nitrogen to the oxygen in the SP(S) form is relatively high $(132.8 \mathrm{~kJ} / \mathrm{mol})$. Simultaneously, there is an elongation of the $\mathrm{C}-\mathrm{O}$ bond in the six-membered ring [1.534 $\AA$ as compared to $1.384 \AA$ for SP(S)]. In fact, when we tried to optimise a hypothetical SP structure that was protonated at the oxygen atom, the structure relaxed to the merocyanine form. This means the only barrier to overcome in this process is that for the proton migration, while there is no additional barrier associated with ringopening. This confirms previous computational studies on a related system. ${ }^{42}$ The barrier for the formation of $\mathrm{SP}(\mathrm{S})$ from the CCC merocyanine form is $164.1 \mathrm{~kJ} / \mathrm{mol}$.

\subsection{Arrival time distributions, collisional isomeriza- tion and photoisomerization of $\mathrm{MCH}^{+} / \mathrm{SPH}^{+}$}

In this section we present the ion mobility data for electrosprayed $\mathrm{MCH}^{+} / \mathrm{SPH}^{+}$ions, demonstrating that the ions can be classified into XTX, XCX and $\mathrm{SPH}^{+}$families which can be selectively interconverted through collisions or photo-excitation. For creating $\mathrm{MCH}^{+} / \mathrm{SPH}^{+}$ ions, spiropyran solution $\left(10^{-4} \mathrm{M}\right.$ in $\left.1: 1 \mathrm{H}_{2} \mathrm{O} / \mathrm{MeOH}\right)$ was injected into the electrospray from a $20 \mathrm{~mL}$ glass syringe at a flow rate of $5 \mu \mathrm{l} / \mathrm{min}$. We relied on the $\mathrm{pH}$ drop occurring in the electrospray process to ensure protonation of SP/MC and no extra acid was added to the solution. The electrosprayed solution was normally irradiated within the syringe for the duration of the experiment using either visible light at $460 \mathrm{~nm}$ to promote formation of SP, or ultraviolet light at $360 \mathrm{~nm}$ to promote formation of MC in solution. ${ }^{38}$ 


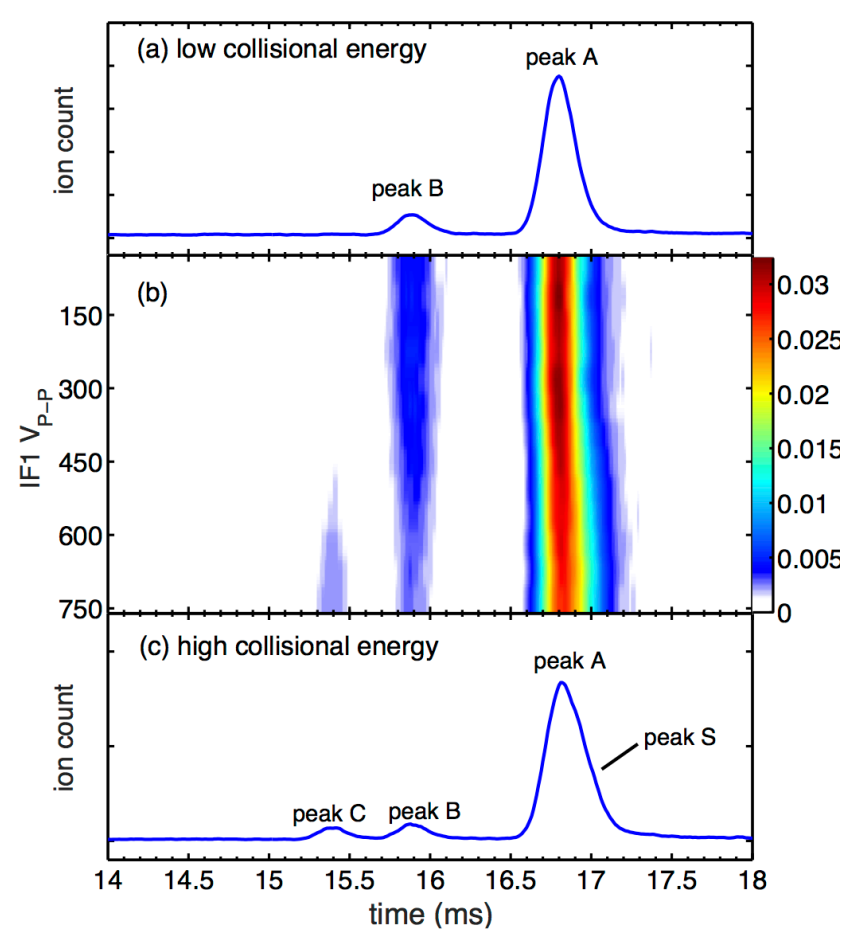

Fig. 5 ATDs recorded with pretreatment of the SP/MC solution with UV light. Plots (a) and (c) show ATDs at low and high collision energy in IF1, respectively. The centre panel (b) represents the ATD as the IF1 RF drive voltage is increased. Note that the total ion count is normalised for each IF1 drive voltage.

Calculated collision cross sections for each conformer with $\mathrm{N}_{2}$ are listed in Table 1 . The cross sections follow a similar trend to those reported by Rogers et al., ${ }^{49}$ for collisions with He. Generally, the XTX $\mathrm{MCH}^{+}$isomers are predicted to have larger collision cross sections (187.4$189.3 \AA^{2}$ ) than the XCX MCH${ }^{+}$and $\mathrm{SPH}^{+}$isomers, which have cross sections in the 173.8-184.4 $\AA^{2}$ and 182.1-184.9 $\AA^{2}$ ranges, respectively.

Figures 5 and 6 show ATDs for $\mathrm{SPH}^{+} / \mathrm{MCH}^{+}$ions generated from solutions that were irradiated with UV or visible light, pretreatments that favour MC or SP, respectively. The relative isomer populations depend on the amplitude of the RF drive voltage for the first ion funnel (IF1) linking the electrospray region to the drift region (see Fig. 2). A high RF voltage ensures efficient ion transfer from the electrospray transfer capillary to the drift region but also causes collisional heating and isomerization. The ATDs obtained at low IF1 RF voltage (Figures 5a and $6 a$ ) show that UV pretreatment favours the slower Peak A, whereas the visible pretreatment favours the

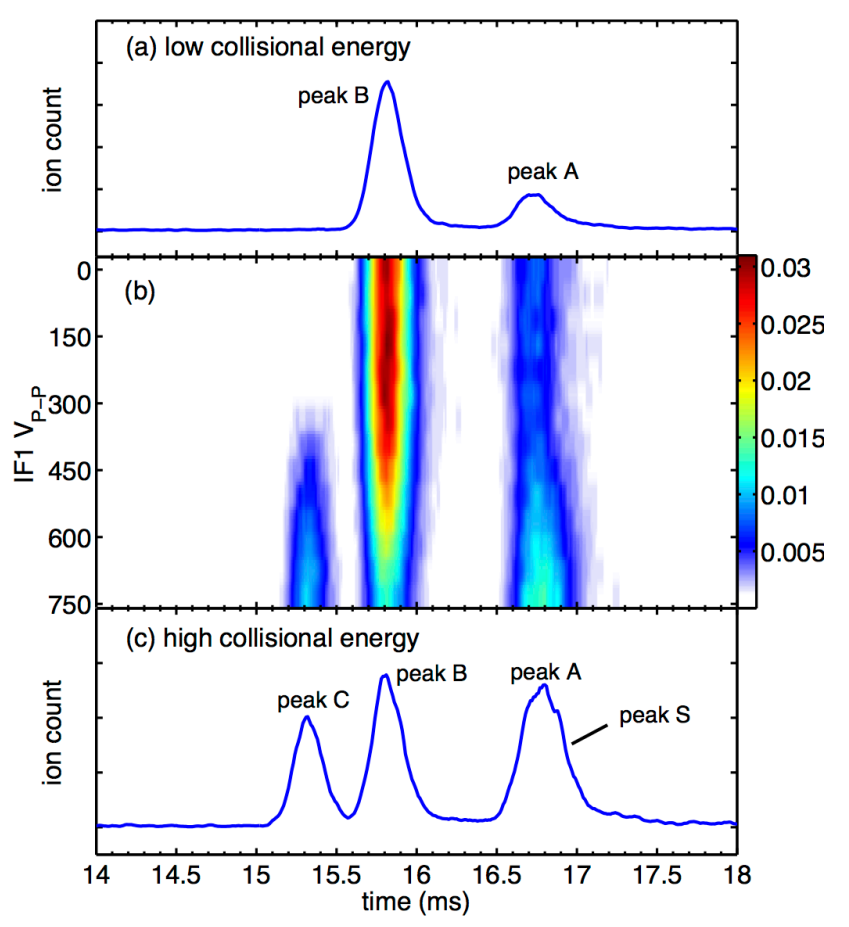

Fig. 6 ATDs recorded with pretreatment of the SP/MC solution with visible light. Plots (a) and (c) show ATDs at low and high collision energy in IF1, respectively. The centre panel (b) represents the ATD as the IF1 RF drive voltage is increased. Note that the total ion count is normalised for each IF1 drive voltage.

faster Peak B. Therefore, one might suppose that peak A corresponds to an extended form of $\mathrm{MCH}^{+}$(predicted to have a large collision cross section) and that peak $\mathrm{B}$ corresponds to $\mathrm{SPH}^{+}$, or to a compact form of $\mathrm{MCH}^{+}$ (both predicted to have smaller collision cross sections). Development of the ATDs with increasing collision energy in IF1 is illustrated in Figs. 5(b) and 6(b). Increasing the IF1 RF voltage leads to transformation of isomer B to an even faster isomer, $\mathrm{C}$, and isomer B to isomer A. Isomer $\mathrm{A}$, on the other hand does not appear to isomerise easily to B or C, but does seem to form an even slower isomer, $\mathrm{S}$, which appears as a shoulder on peak $\mathrm{A}$. The formation onsets for isomer $\mathrm{C}$ and isomer $\mathrm{S}$ in Figs. 5 and 6 suggest that they are formed directly from isomer $B$ and isomer A, respectively. The ATDs recorded at high IF1 RF voltage, shown in Figures 5(c) and 6(c), are very similar to those reported by Rogers et al., ${ }^{49}$ although the mobility resolution was somewhat lower in the earlier study and Peak $\mathrm{S}$ was not resolved.

To help assign peaks $\mathrm{A}, \mathrm{B}, \mathrm{C}$ and $\mathrm{S}$ to specific $\mathrm{MCH}^{+}$ or $\mathrm{SPH}^{+}$isomers we examined their photoresponse us- 


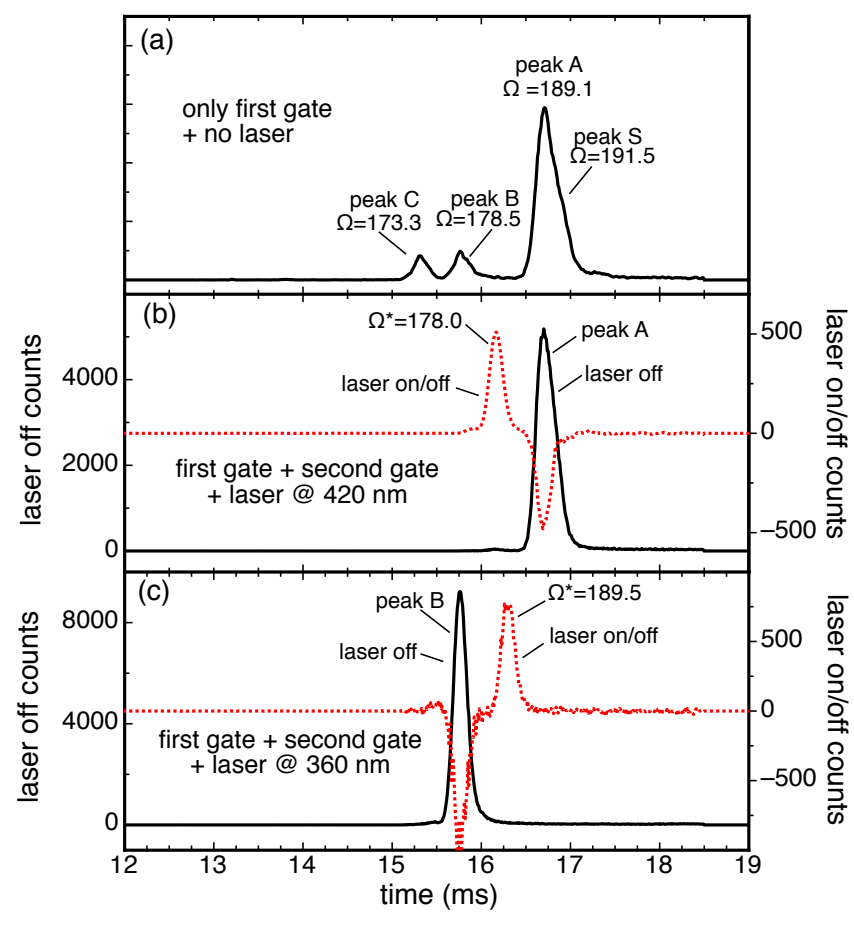

Fig. 7 ATDs for $\mathrm{SPH}^{+} / \mathrm{MCH}^{+}$ions. (a) ATD for a packet of ions with UV pretreatment to favour $\mathrm{MCH}^{+}$ions. (b) ATD with second ion gate timed to pass isomer A. The laser on/off difference trace (dotted red curve) shows photo conversion of isomer A to isomer B at $\lambda=420 \mathrm{~nm}$. (c) ATD with second ion gate timed to pass isomer $\mathrm{B}$. The laser on/off difference trace (dotted red curve) shows photo conversion of isomer B to isomer $\mathrm{A}$ at $\lambda=360 \mathrm{~nm}$. The wavelength dependence for the photo conversion is shown in Figure 8. Measured collision cross sections with $\mathrm{N}_{2}$ are shown for each gated peak $\left(\Omega, \AA^{2}\right)$ and also for photoisomer peaks $\left(\Omega^{*}, \AA^{2}\right)$. Estimated relative uncertainties in cross sections are $\pm 0.3 \AA^{2}$ for parent peaks and $\pm 0.5 \AA^{2}$ for photoisomers.

ing the IMS-photo-IMS approach, irradiating mobilityselected isomers immediately after the second ion gate (IG2) in the drift region (see Figure 2). Photoisomers are separated in the second part of the drift region (after IG2), allowing their identification. ATDs obtained with IG2 timed to transmit either isomer $\mathrm{A}$ or isomer $\mathrm{B}$ are shown in Figures 7(b) and (c) from which it can be seen that isomer $\mathrm{A}$ is depleted and converted to isomer $\mathrm{B}$ by exposure to light at $420 \mathrm{~nm}$, whereas isomer B is transformed to isomer $A$ by light at $360 \mathrm{~nm}$. In both cases a minor amount of isomer $\mathrm{C}$ is also formed. Note, that photo-depletion of one isomer is balanced by photo-enhancement of the other isomers to within $2 \%$, proving that photoisomerization is the dominant process and that photodissociation is insignificant.
By plotting the photo-enhancement (or photo-depletion) signal as a function of wavelength one can generate a photoisomerization action (PISA) spectrum, reflecting the species' absorption spectrum convoluted with a wavelength-dependent photoisomerization yield. PISA spectra for isomers $\mathrm{A}$ and $\mathrm{B}$, along with calculated electronic spectra (from TDA-DFT calculations described in Section 4.1) for isomers of $\mathrm{MCH}^{+}$and $\mathrm{SPH}^{+}$are shown in Fig. 8. Clearly, the photoresponse curve of isomer A, which has a maximum at $400 \mathrm{~nm}$, matches the predicted TDA-DFT absorption spectra of the XTX $\mathrm{MCH}^{+}$isomers (Figure 8) and also the observed spectrum of trans $\mathrm{MCH}^{+}$ in solution which has a maximum at $405 \mathrm{~nm} .{ }^{47}$ On the other hand, isomer B's photoresponse curve corresponds to the predicted absorption spectra of the $\mathrm{XCX} \mathrm{MCH}^{+}$ isomers, and is shifted to shorter wavelength in the near$\mathrm{UV}$, presumably because the electronic conjugation is disrupted by the twisted molecular geometry.

We selected and irradiated isomer $\mathrm{C}$, but could detect neither photoisomerization nor photodissociation over the $300-700 \mathrm{~nm}$ range. Because isomer $C$ seems to be formed collisionally from isomer $\mathrm{B}\left(\mathrm{XCX} \mathrm{MCH}^{+}\right)$in the first ion funnel and because it is not depleted by $300-$ $700 \mathrm{~nm}$ light, it is tempting to assign it to an $\mathrm{SPH}^{+}$isomer (or rapidly interconverting $\mathrm{SPH}^{+}$isomers), that are predicted to absorb light strongly at $220 \mathrm{~nm}$ (see Fig. 8), and to have similar collision cross sections to $\mathrm{XCX} \mathrm{MCH}^{+}$ isomers. Assignment of isomer $\mathrm{C}$ to $\mathrm{SPH}^{+}$is further supported by the fact that its formation threshold from XCX isomers (isomer $\mathrm{B}$ ) appears to be similar to the formation threshold for isomer A (XTX isomers, see Fig. 6), consistent with the computed isomerization barriers (both $\approx 160 \mathrm{~kJ} / \mathrm{mol}$, see Table 1 ).

An alternative assignment for isomer $\mathrm{C}$ is that it is an $\mathrm{XCX} \mathrm{MCH}{ }^{+}$isomer with a small collision cross section, and which for some reason has a low photoisomerization yield. Although we consider it unlikely, it is possible that following photoexcitation that one or more XCX $\mathrm{MCH}^{+}$isomers do not isomerise around the central $\beta$ bond but rather are deactivated by some other means such as fluorescence. The CCT isomer is predicted to be the lowest energy XCX isomer and to have the smallest collision cross section $\left(173.8 \AA^{2}\right)$. The barrier between the CCC and CCT isomers is relatively low $(12 \mathrm{~kJ} / \mathrm{mol}$ at the DSD-PBEP86-D3(BJ)/def2-QZVP level) so that one might expect rapid interconversion of the two forms in both solution and drift region of the IMS-photo-IMS.

It is difficult to isolate isomer $\mathrm{S}$ from isomer $\mathrm{A}$ using IG2 in order to investigate its photoisomerization behaviour selectively. However, at this stage it appears that its photoisomerization cross section is much less than for isomer A over the $300-700 \mathrm{~nm}$ range. Furthermore, 
isomer $\mathrm{S}$ does not appear to be a significant photoisomerization product from either isomer A or isomer B. One possibility is that peak A corresponds to TTT and CTT isomers which interconvert over $\approx 25 \mathrm{~kJ} / \mathrm{mol}$ barriers, whereas peak $\mathrm{S}$ corresponds to TTC and CTC isomers, interconverting over $\approx 20 \mathrm{~kJ} / \mathrm{mol}$ barriers. Interconversion between these XTT and XTC families requires rotation around the $\gamma$ bond, which requires more energy $(\geq 30 \mathrm{~kJ} / \mathrm{mol})$, and only occurs when IF1 RF voltage is increased. A more reliable picture of isomer S's photochemical behaviour will hopefully emerge from future studies using IMS-photo-IMS machines with higher mobility resolution that can properly discriminate isomers A and S.

\section{Discussion}

The quantum-chemical calculations and IMS-photo-IMS investigations provide new insights into the properties of $\mathrm{MCH}^{+}$and $\mathrm{SPH}^{+}$in the gas phase. We can confidently assign the two main peaks, A and B, to XTX and XCX $\mathrm{MCH}^{+}$isomers, respectively, that can be interconverted through photo-excitation. We are also reasonably sure that isomer $\mathrm{C}$, which does not photoisomerize over the 300-700 nm range, can be assigned to $\mathrm{SPH}^{+}$, although we cannot entirely discount the possibility that it is an XCX $\mathrm{MCH}^{+}$isomer that does not undergo photoisomerization following photo-excitation. Interestingly, isomer $C$ is not formed directly from weakly acidic solution but is formed collisionally from $\mathrm{XCX} \mathrm{MCH}^{+}$in the first ion funnel. Isomer $\mathrm{S}$, which was not observed in the previous studies, appears to arise from one or more interconverting XTX isomers, with the simplest explanation being that the A and $S$ families differ by having the $\gamma$ bond in either a trans or cis configuration. The reasons for isomer S's lower photoisomerization yield are currently unclear, and we can only speculate that the excited state dynamics for isomer $\mathrm{S}$ are less efficient in transferring the population through a conical intersection associated with torsion about the $\beta$ bond to form XCX isomers. Generally, our assignments of peaks A, B and C agree with those of Rogers et al. ${ }^{49}$

A significant finding from this investigation is that photoisomerization of $\mathrm{MCH}^{+}$in the gas phase is selective, connecting the $\mathrm{XCX}$ and $\mathrm{XTX}$ isomers, and occurring around the central $\beta C=C$ bond. This suggests that rearrangement is initiated on the excited state PES rather than on the ground state surface after internal conversion to give highly vibrationally excited molecules. If the latter situation prevailed, photo-excitation of a particular isomer would lead to formation of several energetically

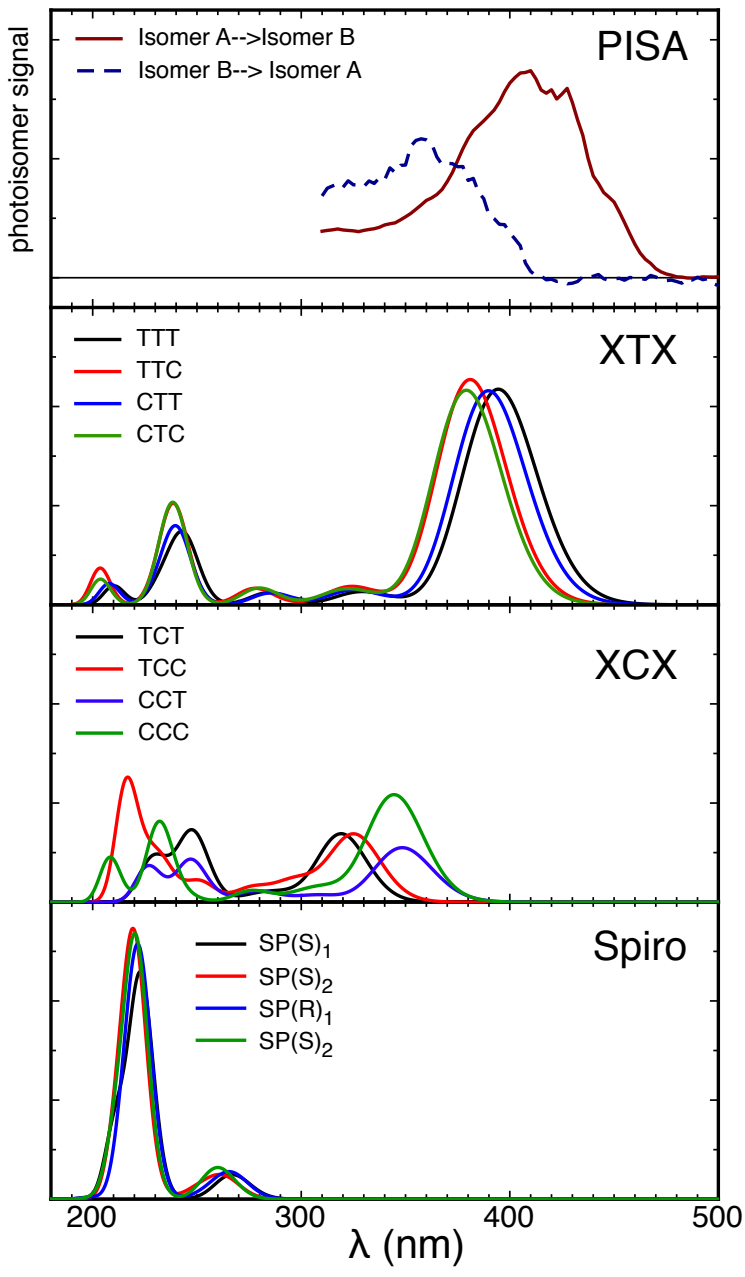

Fig. 8 TDA-DFT electronic spectra for protonated $\mathrm{MCH}^{+}$and $\mathrm{SPH}^{+}$isomers (TDA-B2GPPLYP/def2-TZVP level of theory). Simulated spectra for the merocyanine isomers are grouped by the conformation of the central $\mathrm{C}=\mathrm{C}$ bond (cis or trans). Measured photoisomerization action (PISA) spectra of isomers $\mathrm{A}$ and $\mathrm{B}$ are shown in the top panel. 


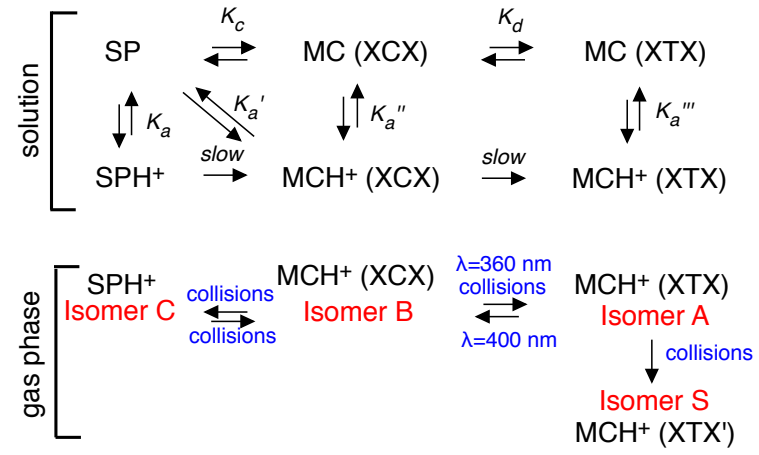

Fig. 9 Scheme for transformations of $\mathrm{SP}, \mathrm{SPH}^{+}, \mathrm{MC}$ and $\mathrm{MCH}^{+}$in solution and gas phase. For the gas phase, the observed processes are indicated.

accessible photo-isomers. For example, although the ion funnel scan shown in Figure 6(b) suggests that $\mathrm{SPH}^{+}$(isomer $\mathrm{C}$ ) is formed from $\mathrm{XCX} \mathrm{MCH}{ }^{+}$(isomer B) at lower collision energy than XTX $\mathrm{MCH}^{+}$(isomer A), photoexcitation of XCX $\mathrm{MCH}^{+}$(isomer B) leads almost exclusively to XTX $\mathrm{MCH}^{+}$(isomer A) with formation of only minor amounts of $\mathrm{SPH}^{+}$(isomer C).

To provide more information on the isomer landscape and complement the IMS-photo-IMS studies it would be desirable to collisionally activate mobility-selected isomers using an IMS-collision-IMS arrangement as demonstrated by Clemmer and coworkers. ${ }^{27,28}$ This would enable one to decide whether isomer $C$ is collisionally converted to $\mathrm{XCX} \mathrm{MCH}^{+}$isomers, as expected if it corresponds to $\mathrm{SPH}^{+}$, and whether isomer $\mathrm{S}$ is converted to isomer A at relatively low collision energies, which should be the case if they correspond to XTT and XTC families.

The gas-phase results have a bearing on the photochemical and isomerization behaviour of SP/MC in weakly acidic solutions. Proposed transformations of $\mathrm{SPH}^{+} / \mathrm{MCH}^{+}$are summarised in Fig. 9. Interestingly, even when conditions are optimised for SP in solution (i.e. visible pre-irradiation of the electrosprayed solution), the most abundant electrosprayed isomer appears to be the XCX form of $\mathrm{MCH}^{+}$(isomer B) rather than $\mathrm{SPH}^{+}$(isomer $C$ ). This suggests that under weak acidic conditions, SP converts directly to an XCX isomer of $\mathrm{MCH}^{+}$rather than forming $\mathrm{SPH}^{+}$. This is consistent with preferential protonation of $\mathrm{SP}$ on the $\mathrm{O}$ atom, leading to immediate ring opening and formation of $\mathrm{XCX} \mathrm{MCH}^{+}$, rather than on the neighbouring $\mathrm{N}$ atom (i.e. $K_{a}>K_{a}^{\prime}$ in Figure 9). Almost 40 years ago, Menger and Perinis postulated that $\mathrm{XCX} \mathrm{MCH}^{+}$was formed directly from protonation of SP to explain the acid catalysed equivalence of the magneti- cally non-equivalent gem-dimethyl groups in SP. ${ }^{48}$ Once formed in solution $\mathrm{XCX} \mathrm{MCH}^{+}$can deprotonate, forming $X C X M C$, which reverts in an almost barrierless reaction to $\mathrm{SP}_{,}^{42}$ or alternatively isomerizes around the $\beta$ bond to form $\mathrm{XTX} \mathrm{MCH}^{+}$. However, the latter process will be extremely slow because of the substantial torsional barrier about the $\beta$ bond $(\approx 160 \mathrm{~kJ} / \mathrm{mol})$. Therefore, a weakly acidified SP solution should initially contain mainly SP and $\mathrm{XCX} \mathrm{MCH}{ }^{+}$. This is consistent with the observations of Wojtyk et al., ${ }^{47}$ who monitored formation of $\mathrm{MCH}^{+}$ from SP in acid solution by observing the growth of its absorption band at $405 \mathrm{~nm}$, which corresponds to XTX $\mathrm{MCH}^{+}$. The conversion rate was found to have an inverse dependence on $\left[\mathrm{H}^{+}\right]$, an observation that was rationalised through a kinetic scheme in which $\mathrm{SPH}^{+}$was an unreactive sink that prevented formation of $\mathrm{MCH}^{+}$. The fact that $\mathrm{SPH}^{+}$is not produced directly from the electrosprayed solution but only after collisions of XCX $\mathrm{MCH}^{+}$in the first ion funnel, suggests that, at least in weakly acidic solution, it is actually the $\mathrm{XCX} \mathrm{MCH}{ }^{+}$isomer that mainly serves as an unreactive reservoir for $\mathrm{SP}$ $\left(\right.$ rather than $\mathrm{SPH}^{+}$), and which prevents its conversion to the more stable XTX $\mathrm{MCH}^{+}$isomer. However, the XCX $\mathrm{MCH}^{+}$isomer absorbs light at almost the same wavelength as neutral SP making it difficult to distinguish the two isomers from UV/Vis spectra.

The current study highlights the quite different behaviours of neutral and protonated SP/MC. In particular, the barriers for rotation around the $\alpha, \beta$ and $\gamma$ bonds are quite different for the neutral $\mathrm{MC}$ and protonated $\mathrm{MCH}^{+}$ species. Neutral MC has quinoidal and zwitterionic resonance forms and consequently the 3 polyene bonds have mixed single/double bond character, ${ }^{48}$ reflected in transcis isomerization barriers lying in the $50-70 \mathrm{~kJ} / \mathrm{mol}$ range for all 3 bonds. ${ }^{42}$ In contrast, the $\mathrm{MCH}^{+}$ion, which is protonated on the $\mathrm{O}$ atom has a clear single-double-single bond character for the polyene chain, with trans-cis isomerization barriers of $20-35 \mathrm{~kJ} / \mathrm{mol}$ for the $\alpha$ and $\gamma$ bonds and $140-170 \mathrm{~kJ} / \mathrm{mol}$ for the central $\beta$ double bond. Therefore the XCX MCH ${ }^{+}$isomers end up being separated from the XTX $\mathrm{MCH}^{+}$and SP isomers by substantial barriers, whereas, in contrast, the neutral CCC MC isomer, for example, should be short lived because it is separated from the much more stable SP isomer by only a small barrier $(\approx 5 \mathrm{~kJ} / \mathrm{mol})$ and from other $\mathrm{XCX}$ isomers by $\approx 60 \mathrm{~kJ} / \mathrm{mol}$ barriers. ${ }^{42}$

\section{Summary and Outlook}

The main outcomes of our computational and experimental studies on the protonated spiropyran/merocyanine 
system can be summarised as follows.

1. XTX and XCX isomers of $\mathrm{MCH}^{+}$are both stable in the gas phase and can be interconverted through isomerization around the $\mathrm{C}=\mathrm{C}$ double bond by light or through collisions. The $\mathrm{XCX} \mathrm{MCH}^{+}$isomers absorb light at substantially shorter wavelength than XTX $\mathrm{MCH}^{+}$isomers, with maxima in their response curves at $360 \mathrm{~nm}$ and $400 \mathrm{~nm}$, respectively.

2. Although $\mathrm{SPH}^{+}$isomers are not produced directly in the gas phase by electrospraying weakly acidic solutions, they can be formed from collisional excitation of XCX $\mathrm{MCH}^{+}$isomers.

3. Photoisomerization is more selective than collisional isomerization for the $\mathrm{SPH}^{+} / \mathrm{MCH}^{+}$system. Whereas, collisions can be used to transform between any two isomers (albeit through intermediates), photoconversion between XTX and $\mathrm{XCX} \mathrm{MCH}^{+}$isomers occurs selectively through rotation about the $\beta \mathrm{C}=\mathrm{C}$ bond, with practically no formation of $\mathrm{SPH}^{+}$.

4. Controlled collisional measurements using a tandem IMS-collision-IMS arrangement, as developed by Clemmer and coworkers, ${ }^{27,28}$ should enable determination of isomerization barriers for the $\mathrm{MCH}^{+}$ and $\mathrm{SPH}^{+}$isomers, allowing direct comparisons with dispersion-corrected DFT calculations described in this paper.

5. Care is necessary to avoid collision induced isomerization in regions of high electric field prior to photoexcitation. The possibility for collision induced isomerization in an ion funnel is exemplified by the results shown in Figure 6 where $\mathrm{XCX} \mathrm{MCH}{ }^{+}$ isomers are transformed to $\mathrm{SPH}^{+}$isomers at intermediate drive voltages for the first ion funnel.

Undoubtedly, it would be useful to examine $\mathrm{MCH}^{+} / \mathrm{SPH}^{+}$ isomers using other forms of gas-phase ion spectroscopy. For example, it should be feasible to distinguish and spectroscopically characterize some of the key isomers using double-resonance IR-IR or IR-Vis, IR/UV strategies involving vibrational and electronic excitation. ${ }^{7-9}$ The $\mathrm{NH}$ and $\mathrm{OH}$ stretch bands in the IR spectrum should serve as clear signatures for the presence of $\mathrm{SPH}^{+}$and $\mathrm{MCH}^{+}$ isomers, respectively.

More generally, this paper outlines continuing developments in combining ion mobility spectrometry and laser spectroscopy. Investigations of the $\mathrm{SPH}^{+} / \mathrm{MCH}^{+}$ system and of carbocyanine dyes ${ }^{14}$ illustrate the utility of the tandem IMS-photo-IMS approach for identifying and characterising isomeric forms of charged, photoactive molecules. The IMS-photo-IMS approach alleviates ambiguities concerning the identity of the absorbing isomer, and is ideally suited for spectroscopically probing systems that have high dissociation energies but low isomerization barriers. For these reasons, it should eventually be adopted more broadly to complement traditional approaches for spectroscopically characterizing molecular ions. Future developments are likely to revolve around utilizing IMS stages with higher mobility resolution, allowing better isomer discrimination and deploying combinations of IMS and photodissociation spectroscopy.

\section{Acknowledgements}

This research was supported under the Australian Research Council's Discovery Project funding scheme (Project Numbers DP110100312 and DP120100100). LG is the recipient of an Australian Research Council Discovery Early Career Researcher Award (Project Number DE140100550). LG also acknowledges funding from the Selby Scientific Foundation through the 2014 Selby Research Award and generous allocation of computing time from the National Computational Infrastructure (NCI) National Facility within the National Computational Merit Allocation Scheme (Project fk5). We thank Professor Matthew Bush for providing a modified version of the MOBCAL code with $\mathrm{N}_{2}$ collision parameters described in ref. 25 .

\section{References}

1 M. A. Duncan, Int. J. Mass Spectrom., 2000, 200, 545-569.

2 E. J. Bieske and J. P. Maier, Chem. Revs., 1993.

3 E. J. Bieske and O. Dopfer, Chem. Revs., 2000, 100, 3963.

4 N. C. Polfer and J. Oomens, Mass Spectrom. Rev., 2009, 28, 468-494.

5 R. C. Dunbar, Int. J. Mass Spectrom., 2014, 377, 159-171.

6 Y. Koyama, K. Kubo, M. Komori, H. Yasuda and Y. Mukai, Photochem. Photobiol., 1991, 54, 433-443.

7 T. R. Rizzo, J. A. Stearns and O. V. Boyarkin, Int. Rev. Phys. Chem., 2009, 28, 481-515.

8 N. L. Burke, J. G. Redwine, J. C. Dean, S. A. McLuckey and T. S. Zwier, Int. J. Mass Spectrom., 2014, 378, 196-205.

9 A. Wolk, C. Leavitt, E. Garand and M. Johnson, Acc. Chem. Res., 2014, 47, 202-210.

10 B. D. Adamson, N. J. A. Coughlan, R. Continetti and E. J. Bieske, Phys. Chem. Chem. Phys., 2013, 15, 9540 - 9548.

11 B. D. Adamson, N. J. A. Coughlan, G. da Silva and E. J. Bieske, J. Phys. Chem. A, 2013, 117, 13319-13325.

12 N. J. A. Coughlan, K. J. Catani, B. D. Adamson, U. Wille and E. J. Bieske, J. Chem. Phys., 2014, 140, 164307.

13 N. J. A. Coughlan, B. D. Adamson, K. J. Catani, U. Wille and E. J. Bieske, J. Phys. Chem. Lett., 2014, 5, 3195-3199.

14 B. D. Adamson, N. J. A. Coughlan, P. B. Markworth, R. E. Continetti and E. J. Bieske, Rev. Sci. Instrum., 2014, 85, 123109. 
15 G. A. Eiceman, Z. Karpas and H. H. Hill, Jr., Ion Mobility Spectrometry, CRC Press, Boca Raton, 3rd edn, 2014.

16 A. B. Kanu, P. Dwivedi, M. Tam, L. Matz and H. H. Hill Jr, J. Mass Spec., 2008, 43, 1.

17 T. Wyttenbach, N. A. Pierson, D. E. Clemmer and M. T. Bowers, Ann. Rev. Phys. Chem., 2014, 65, 175-196.

18 R. Cumeras, E. Figueras, C. E. Davis, J. I. Baumbach and I. Gràcia, Analyst, 2015, 140, 1376-1390.

19 R. Cumeras, E. Figueras, C. E. Davis, J. I. Baumbach and I. Gràcia, Analyst, 2015, 140, 1391-1410.

20 C. Wu, W. F. Siems, G. R. Asbury and H. H. Hill, Anal. Chem., 1998, 70, 4929-4938.

21 P. Dugourd, R. R. Hudgins, D. E. Clemmer and M. F. Jarrold, Rev. Sci. Instr., 1997, 68, 1122.

22 T. Wyttenbach and M. T. Bowers, Ann. Rev. Phys. Chem., 2007, 58, 511.

23 E. S. Baker, B. H. Clowers, F. Li, K. Tang, A. V. Tolmachev, D. C. Prior, M. E. Belov and R. D. Smith, J. Am. Soc. Mass Spectrom., 2007, 18, 1176-1187.

24 S. I. Merenbloom, R. S. Glaskin, Z. B. Henson and D. E. Clemmer, Anal. Chem., 2009, 81, 1482-1487.

25 I. Campuzano, M. F. Bush, C. V. Robinson, C. Beaumont, K. Richardson, H. Kim and H. I. Kim, Anal. Chem., 2012, 84, 1026-1033.

26 S. L. Koeniger, S. I. Merenbloom, S. J. Valentine, M. F. Jarrold, H. R. Udseth, R. D. Smith and D. E. Clemmer, Anal. Chem., 2006, 78, 41614174 .

27 N. A. Pierson, S. J. Valentine and D. E. Clemmer, J. Phys. Chem. B, 2010, 114, 7777-7783.

28 N. A. Pierson and D. E. Clemmer, Int. J. Mass Spectrom., 2014, 646654.

29 K. Koyasu, T. Ohtaki, N. Hori and F. Misaizu, Chem. Phys. Lett., 2012, $523,54-59$.

30 S. M. Zucker, S. Lee, N. Webber, S. J. Valentine, J. P. Reilly and D. E. Clemmer, J. Am. Soc. Mass Spectrom., 2011, 22, 1477-1485.

31 S. Warnke, C. Baldauf, M. T. Bowers, K. Pagel and G. von Helden, J. Am. Chem. Soc., 2014, 136, 10308-10314.

32 B. Bellina, J. M. Brown, J. Ujma, P. Murray, K. Giles, M. Morris, I. Compagnon and P. E. Barran, Analyst, 2014, 139, 6348-6351.

33 O. Hernandez, S. Isenberg, V. Steinmetz, G. L. Glish and P. Maître, J. Phys. Chem. A, 2015, in press, DOI: 10.1021/jp511975f.

34 G. Papadopoulos, A. Svendsen, O. V. Boyarkin and T. R. Rizzo, J. Am. Soc. Mass. Spectrom., 2012, 23, 1173-1181.

35 S. Warnke, J. Seo, J. Boschmans, F. Sobott, J. H. Scrivens, C. Bleiholder, M. T. Bowers, S. Gewinner, W. Schollkopf, K. Pagel and G. von Helden, J. Am. Chem. Soc., 2015, in press, DOI: $10.1021 /$ jacs.5b01338.

36 H. E. Revercomb and E. A. Mason, Anal. Chem., 1975, 47, 970-983.

37 J. C. May, C. R. Goodwin, N. M. Lareau, K. L. Leaptrot, C. B. Morris, R. T. Kurulugama, A. Mordehai, C. Klein, W. Barry, E. Darland, G. Overney, K. Imatani, G. C. Stafford, J. C. Fjeldsted and J. A. McLean, Anal. Chem., 2014, 86, 2107-2116.

38 R. Klajn, Chem. Soc. Rev., 2013, 43, 148.

39 X. Xie and E. Bakker, J. Am. Chem. Soc., 2014.

40 Y. Futami, M. Chin, S. Kudoh and M. Takayanagi, Chem. Phys., 2003, 370, 460-468.

41 G. Cottone, R. Noto and G. La Manna, Chem. Phys. Lett., 2004, 388, 218-222.

42 R. Ganesan and F. Remacle, Theor. Chem. Acc., 2012, 131, 1255.

43 S. Ruetzel, M. Diekmann, P. Nuernberger, C. Walter, B. Engels and T. Brixner, J. Chem. Phys., 2014, 140, 224310.

44 A.-K. Holm, M. Rini, E. T. J. Nibbering and H. Fidder, Chem. Phys. Lett., 2003, 376, 214-219.
45 C. J. Wohl and D. Kuciauskas, J. Phys. Chem. B, 2005, 109, 21893 21899.

46 A. Gahlmann, I.-R. Lee and A. H. Zewail, Angew. Chemie Int. Ed., 2010, 49, 6524-6527.

47 J. T. C. Wojtyk, A. Wasey, N.-N. Xiao, P. M. Kazmaier, S. Hoz, C. Yu, R. P. Lemieux and E. Buncel, J. Phys. Chem. A, 2007, 111, 2511-2516.

48 F. M. Menger and M. Perinis, Tetrahedron Letters, 1978, 19, 4653-4656.

49 R. A. Rogers, A. R. Rodier, J. A. Stanley, N. A. Douglas, X. Li and W. J. Brittain, Chem. Commun., 2014, 50, 3424-3426.

50 F. Neese, Wiley Interdiscip. Rev.: Comput. Mol. Sci., 2012, 2, 73-78.

51 Y. Zhao and D. G. Truhlar, J. Phys. Chem. A, 2005, 109, 5656-5667.

52 S. Grimme, J. Antony, S. Ehrlich and H. Krieg, J. Chem. Phys., 2010, 132, 154104.

53 S. Grimme, S. Ehrlich and L. Goerigk, J. Comput. Chem., 2011, 32, 1456-1465.

54 F. Weigend and R. Ahlrichs, Phys. Chem. Chem. Phys., 2005, 7, 32973305.

55 S. Kozuch and J. M. L. Martin, Phys. Chem. Chem. Phys., 2011, 13, 20104-20107.

56 J. Perdew and K. Schmidt, In: Density Functional Theory and Its Applications to Materials., Melville, NY, 2001, pp. 1-20.

57 S. Grimme, J. Chem. Phys., 2006, 124, 034108.

58 L. Goerigk and S. Grimme, Wiley Interdiscip. Rev.: Comput. Mol. Sci., 2014, 4, 576-600.

59 L. Goerigk and S. Grimme, J. Chem. Theory Comput., 2010, 6, 107-126.

60 L. Goerigk and S. Grimme, J. Chem. Theory Comput., 2011, 7, 291-309.

61 L. Goerigk and S. Grimme, Phys. Chem. Chem. Phys., 2011, 13, 66706688.

62 A. D. Becke, J. Chem. Phys., 1993, 98, 5648-5652.

63 P. J. Stephens, F. J. Devlin, C. F. Chabalowski and M. J. Frisch, J. Phys. Chem., 1994, 98, 11623-11627.

64 T. Yanai, D. Tew and N. Handy, Chem. Phys. Lett., 2004, 393, 51.

65 A. Karton and L. Goerigk, J. Comput. Chem., 2015, 36, 622-632.

66 S. Grimme and F. Neese, J. Chem. Phys., 2007, 127, 154116.

67 L. Goerigk, J. Moellmann and S. Grimme, Phys. Chem. Chem. Phys., 2009, 11, 4611-4620.

68 L. Goerigk and S. Grimme, J. Chem. Phys., 2010, 132, 184103.

69 L. Goerigk and S. Grimme, J. Chem. Theory Comput., 2011, 7, 32723277.

70 L. Goerigk and S. Grimme, J. Phys. Chem. A, 2009, 113, 767-776.

71 A. L. Fetter and J. D. Walecka, Quantum Theory of Many-Particle Systems, McGraw-Hill, New York, 1971.

72 S. Hirata and M. Head-Gordon, Chem. Phys. Lett., 1999, 314, 291-299.

73 A. Karton, A. Tarnopolsky, J. F. Lamere, G. C. Schatz and J. M. L. Martin, J. Phys. Chem. A, 2008, 112, 12868-12886.

74 M. Mesleh, J. Hunter, A. Shvartsburg, G. Schatz and M. Jarrold, J. Phys. Chem., 1996, 100, 16082-16086.

75 A. A. Shvartsburg and M. F. Jarrold, Chem. Phys. Letts., 1996, 261, 86-91.

76 C. M. Breneman and K. B. Wiberg, J. Comput. Chem., 1990, 11, 361373.

77 D. C. Graham, A. S. Menon, L. Goerigk, S. Grimme and L. Radom, J. Phys. Chem. A, 2009, 113, 9861-9873.

78 R. Peverati and M. Head-Gordon, J. Chem. Phys., 2013, 139, 024110. 


\section{University Library}

\section{- M M N E R VA A gateway to Melbourne's research publications}

Minerva Access is the Institutional Repository of The University of Melbourne

Author/s:

Markworth, PB;Adamson, BD;Coughlan, NJA;Goerigk, L;Bieske, EJ

Title:

Photoisomerization action spectroscopy: flicking the protonated merocyanine-spiropyran switch in the gas phase

Date:

2015-01-01

Citation:

Markworth, P. B., Adamson, B. D., Coughlan, N. J. A., Goerigk, L. \& Bieske, E. J. (2015). Photoisomerization action spectroscopy: flicking the protonated merocyaninespiropyran switch in the gas phase. PHYSICAL CHEMISTRY CHEMICAL PHYSICS, 17 (39), pp.25676-25688. https://doi.org/10.1039/c5cp01567g.

Persistent Link:

http://hdl.handle.net/11343/123632 